\title{
Papel de la tomografía computarizada de tórax en pacientes con neumonía por SARS-CoV-2. Experiencia del Hospital Ángeles Lomas
}

\section{Role of chest CT in SARS-CoV-2 pneumonia patients. Experience of Hospital Angeles Lomas}

\author{
Héctor Manuel Velasco Jiménez, * Francisco René Hernández Ortiz, * \\ Horacio Lozano Zalce, ${ }^{\ddagger}$ Mario Calva Arcos, ${ }^{\ddagger}$ Ángel César Chávez Alanís ${ }^{\ddagger}$ \\ Citar como: Velasco JHM, Hernández OFR, Lozano ZH, Calva AM, Chávez AÁC. Papel de la tomografía \\ computarizada de tórax en pacientes con neumonía por SARS-CoV-2. Experiencia del Hospital \\ Ángeles Lomas. Acta Med. 2020; 18 (4): 390-394. https://dx.doi.org/10.35366/97265
}

\section{Resumen}

Objetivo: Aclarar el papel de la TC en el manejo de pacientes con neumonía por SARS-CoV-2 y generar reportes radiológicos estandarizados. Material y métodos: Se valoraron 108 tomografías de tórax simple de pacientes con sospecha de neumonía por SARS-CoV-2, a quienes además se les realizo RT-PCR. Resultados: 68 pacientes tuvieron hallazgos tomográficos sugestivos de neumonía por SARS-CoV-2 (consensos de la RSNA y CO-RADS), de los cuales 51 tuvieron adicionalmente prueba de RT-PCR positiva. En el otro grupo de 40 pacientes se obtuvieron tomografías normales o negativas, con seis resultados positivos para RT-PCR respectivamente. La sensibilidad de la TC en nuestro estudio fue de $89 \%$, la especificidad de $66 \%$ con una LR (+) de 2.61 y LR (-) de 0.16 . Conclusión: La TC carece de especificidad para considerarse prueba diagnóstica; sin embargo, su uso como herramienta de tamizaje está justificado en ciertos contextos clínicos. Una posible virtud de la TC es su utilidad como herramienta en el pronóstico y por ende, en el manejo de los pacientes.

Palabras clave: Tomografía computarizada, SARS-CoV-2, vidrio despulido, crazy-paving, CO-RADS, RSNA.

\section{Abstract}

Objective: To clarify the role of CT in the management of patients with SARS-CoV-2 pneumonia and to generate standardized radiological reports. Material and methods: 108 simple chest tomography scans were evaluated, of patients with suspected SARS-CoV-2 pneumonia who also underwent RT-PCR. Results: 68 patients had tomographic findings suggestive of SARS-CoV-2 pneumonia (consensus of the RSNA and CO-RADS), of which 51 had additionally positive RT-PCR test. The other group of 40 patients had normal or negative tomographies, with 6 positive results for RT-PCR respectively. The sensitivity of the CT in our study was $89 \%$, the specificity of $66 \%$ with an LR (+) of 2.61 and LR (-) of 0.16 . Conclusion: CT lacks specificity to be considered a diagnostic test, however its use as a screening tool is justified in certain clinical contexts. A potential virtue of CT is its utility as a tool in prognosis and therefore patient management.

Keywords: Computed tomography, SARS-CoV-2, ground glass, crazy-paving, CO-RADS, RSNA.

\footnotetext{
* Médico residente de Imagenología Diagnóstica y Terapéutica, UNAM.

‡ Médico radiólogo adscrito al Servicio de Imagenología.
}

Correspondencia:

Héctor Manuel Velasco Jiménez

Correo electrónico: hector.velasco.md@gmail.com

Aceptado: 27-08-2020. 


\section{INTRODUCCIÓN}

Desde marzo de 2020, cuando la OMS dio a conocer su postura sobre el nuevo coronavirus SARS-CoV-2 y se declaró pandemia por el mismo, en nuestro país se han reportado de manera oficial 74,560 casos con 8,134 muertes confirmadas (al 26 de mayo 2020) relacionadas con el virus SARS-CoV-2. ${ }^{1}$

$\mathrm{Al}$ igual que otros coronavirus respiratorios, el nuevo virus es transmitido principalmente por gotitas a través de la vía respiratoria, con un periodo de incubación antes del comienzo de los síntomas de cuatro a cinco días promedio así como $97.5 \%$ de pacientes sintomáticos a los 11.5 días. ${ }^{2}$

El empleo de protocolos de triaje en los servicios de urgencias y la optimización de recursos disponibles como equipo de protección personal (EPP), pruebas de RT-PCR y TC, por mencionar algunos, son fundamentales para el manejo integral y adecuado de los pacientes con sospecha de COVID-19. Debido a la repercusión epidemiológica que implica realizar el diagnóstico de pacientes con infección por SARS-CoV-2, resulta trascendente acortar los tiempos para la realización del diagnóstico.

La RT-PCR es el estándar de oro para el diagnóstico y por ende, resulta esencial su realización; sin embargo, el aparente rezago en la obtención de los resultados de la misma (seis horas en condiciones ideales), su disponibilidad, sensibilidad (con gran variabilidad, reportada entre $42-71 \%$ en la literatura médica) y los falsos negativos (carga viral baja), contrastan con la relativa rapidez de los obtenidos tras la realización del estudio tomográfico, contribuyendo así a un mayor uso de esta modalidad de imagen médica en el abordaje de estos pacientes.

Actualmente, la mayoría de las sociedades radiológicas en el mundo (incluyendo algunas de las más reconocidas en el continente americano como STR, ACR y RSNA) no recomiendan la TC simple de tórax como herramienta para el tamizaje de pacientes con sospecha de neumonía por SARS-CoV-2.

Muchas han sido las publicaciones radiológicas relacionadas con COVID-19 hasta la fecha, las cuales abarcan desde los hallazgos tomográficos más frecuentes, la evolución de los mismos a través del tiempo, la estimación de la severidad y la estandarización de reportes radiológicos, por mencionar algunos.

Dentro de los estudios enfocados en los hallazgos tomográficos llaman la atención los que concluyen que los patrones radiológicos típicos en pacientes con infección por el virus SARS-CoV-2, son las opacidades en vidrio despulido de predominio periférico, bilateral y multilobar así como el patrón que asemeja neumonía organizada. Sin embargo, también se han reportado patrones en adoquín crazy-paving, opacidades lineales, curvilíneas, perilobula- res, nódulos, consolidación y vidrio despulido difuso que pueden presentarse en otros procesos patológicos incluyendo infecciones por diferentes patógenos, neumopatías por exposición y toxicidad por fármacos, por mencionar algunos. $^{3}$

Por lo antes mencionado, la principal debilidad del estudio tomográfico es la especificidad con rangos que van de $25-53 \%$, mientras que la sensibilidad va de $60-98 \%$ de acuerdo a la literatura médica reciente. Es importante mencionar que aunque no es la única modalidad de imagen que se puede usar para el abordaje de estos pacientes, ya que también están ampliamente disponibles la radiología convencional y el ultrasonido a la cabecera del paciente, ésta es la modalidad de imagen que brinda mayor información y está sujeta a menor cantidad de sesgos.

\section{MATERIAL Y MÉTODOS}

En un periodo comprendido entre el 22 de febrero y el 09 de abril de 2020 se valoró un total de 771 pacientes por sospecha de COVID-19 en el Servicio de Urgencias del Hospital Ángeles Lomas, de los cuales 108 además fueron estudiados por tomografía (Tabla 1).

Para el presente estudio se excluyeron aquellos pacientes con prueba RT-PCR negativa, quedando un total de 57 pacientes con una prueba positiva y tomografía simple con protocolo de alta resolución en equipo LightSpeed VCT GE Medical Systems (64 detectores).

Tipo de estudio: se trata de un estudio observacional y retrospectivo.

\section{RESULTADOS}

Los hallazgos tomográficos en los pacientes con sospecha de COVID-19 fueron categorizados en conjunto por tres médicos radiólogos con experiencias de 24, 14 y cuatro años, así como dos médicos residentes de cuarto y segundo año del curso de especialización en imagenología diagnóstica y terapéutica de acuerdo al consenso de la RSNA/STR/ ACR y el sistema CO-RADS.

De los 108 pacientes con sospecha de infección por SARSCoV-2 estudiados por tomografía, 60 fueron mujeres y 48

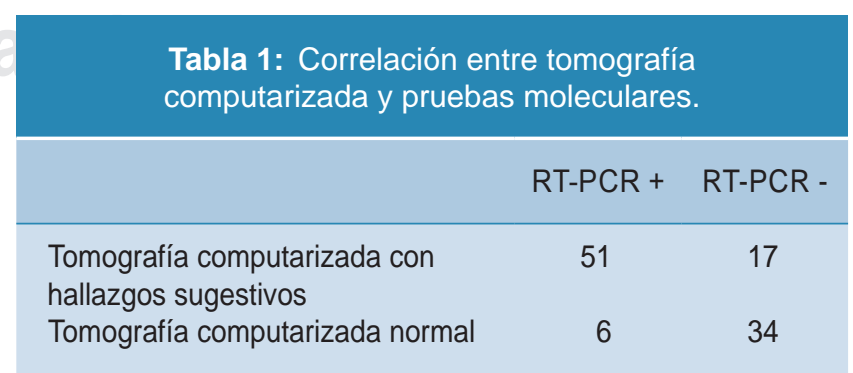


Tabla 2: Declaración del consenso de expertos

RSNA sobre informes en tomografía computarizada

de tórax con hallazgos relacionados con

COVID-19 y su correlación con CO-RADS.

Pacientes

Pacientes

$\begin{array}{lrrr}\text { Negativo } & 6 & \text { CO-RADS 1 } & 6 \\ \text { Atípico } & 2 & \text { CO-RADS 2 } & 2 \\ \text { Indeterminado } & 10 & \text { CO-RADS 3 } & 8 \\ & & \text { CO-RADS 4 } & 2 \\ \text { Típico } & 39 & \text { CO-RADS 5 } & 39\end{array}$

RSNA = Radiological Society of North America .

hombres con una edad promedio de 45 años. El tiempo promedio de evolución con síntomas respiratorios fue de 7.1 días.

De los estudios tomográficos, 40 fueron categorizados como normales o negativos, mientras que 68 reportaron hallazgos sugestivos de afección infecto/inflamatoria.

Se incluyeron 57 pacientes con prueba positiva y estudio tomográfico en el análisis, de los cuales 40 fueron varones y 17 mujeres respectivamente.

En $89 \%$ de nuestros pacientes se documentaron hallazgos tomográficos, siendo categorizados como típicos en $39(68.42 \%)$, indeterminados en 10 (17.54\%), atípicos en dos (3.5\%) y negativos en seis $(10.52 \%)$ de acuerdo al consenso RSNA/STR/ACR. Estas categorías a su vez muestran cierta correlación con el sistema CO-RADS propuesto originalmente por la sociedad radiológica holandesa. ${ }^{4}$ En nuestro estudio los hallazgos típicos del consenso RSNA/ STR/ACR fueron encasillados como CO-RADS 5, los atípicos como CO-RADS 2 y los negativos como CO-RADS 1. Sin embargo, la categoría indeterminada mostró mayor flexibilidad y pudo ser dictaminada como CO-RADS 3 y 4 (Tabla 2).

La sensibilidad del estudio tomográfico en pacientes con COVID-19 en nuestro estudio fue de $89 \%$, con una especificidad de $66 \%$, la cual se encuentra por arriba de lo reportado en la literatura médica reciente.

Debido a que se trata de una enfermedad nueva, la aplicabilidad de "valores predictivos positivos y negativos" está a discusión (ya que no se conoce a ciencia cierta la prevalencia de la enfermedad); sin embargo, una evaluación más robusta y útil en este contexto es el cálculo de la razón de verosimilitud. ${ }^{5}$

$\operatorname{LR}(+)=($ sensibilidad $/ 1$-especificidad $)=0.89 / 1-0.66)$ $=2.61$

$\operatorname{LR}(-)=(1$-sensibilidad/especificidad $)=1-0.89 / 0.66)=0.16$
El valor de LR $(+)$ de 2.6 es considerado "regular", mientras que el valor de LR (-) de 0.16 es considerado "bueno".

De acuerdo a la lógica bayesiana, el mayor beneficio tras la implementación de una prueba diagnóstica se obtiene cuando los individuos tienen una probabilidad intermedia de la enfermedad (20-80\%); ${ }^{6}$ sin embargo, hasta la fecha, no disponemos de un método que ayude a estimar dicha probabilidad "preprueba" de forma objetiva, por lo que la tomografía computarizada tentativamente puede proporcionar información valiosa para el manejo de los pacientes y no sólo corroborar un diagnóstico de neumonía viral.

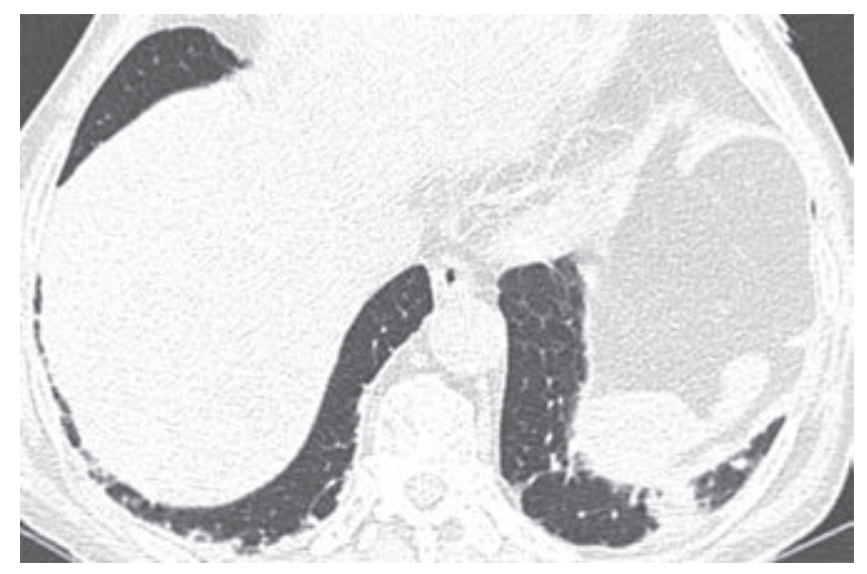

Figura 1: Pequeña zona de consolidación en segmento posterobasal izquierdo sin vidrio despulido asociado: hallazgos tomográficos atípicos consenso RSNA/STR/ACR con categoría equivalente CO-RADS 2.

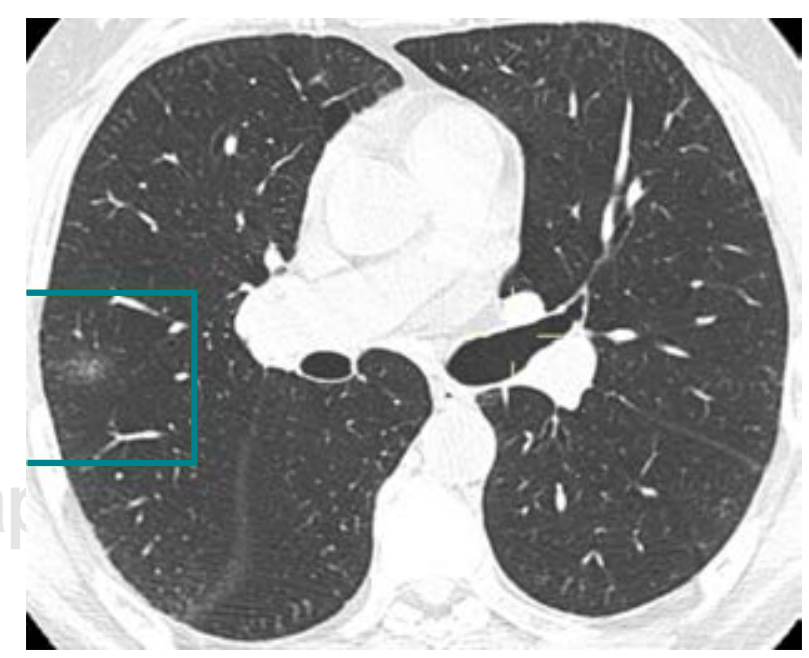

Figura 2: Pequeña zona de vidrio despulido unilateral con distribución no específica y sin consolidación asociada: hallazgos tomográficos indeterminados consenso RSNA/STR/ ACR con categoría equivalente CO-RADS 3. 


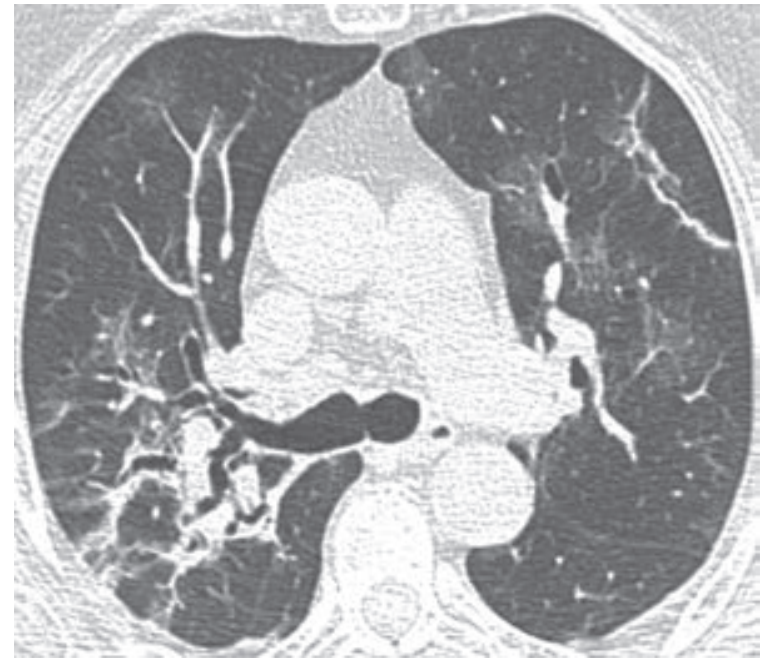

Figura 3: Múltiples infiltrados en vidrio despulido sin distribución específica asociados a consolidación: hallazgos tomográficos indeterminados consenso RSNA/STR/ACR con categoría equivalente CO-RADS 4.

\section{DISCUSIÓN}

El uso de tomografía simple de tórax en pacientes inmunocompetentes con enfermedad aguda del tracto respiratorio así como la exploración física anormal, alteración de signos vitales, enfermedad orgánica cerebral u otros factores de riesgo usualmente es apropiado como siguiente método de estudio por imagen, si la radiografía de tórax es equivocada o negativa de acuerdo al último Consenso del Colegio Americano de Radiología revisado en $2018 .{ }^{7}$ Otros escenarios con pacientes inmunocompetentes, pero con comorbilidades importantes como asma y EPOC de forma similar consideran la tomografía como estudio de imagen de segunda línea.

El Consenso Multinacional de la Sociedad de Fleischner sobre el papel de los estudios de imagen de tórax en el manejo de pacientes durante la actual pandemia pondera la radiología convencional y la tomografía como protagonistas. Considera a la radiografía convencional de poca utilidad en infecciones leves o tempranas, mientras que la tomografía simple muestra más utilidad debido a su mayor sensibilidad en estos contextos, lo cual puede tener implicaciones relevantes en cuestiones de salud pública (aislamiento de pacientes) en situaciones donde los recursos diagnósticos (pruebas moleculares) son limitados. ${ }^{8}$

El seguimiento de los pacientes con neumonía por SARS-CoV-2 puede realizarse mediante radiografías convencionales; sin embargo, es claro que existen escenarios en los cuales la tomografía puede brindar información valiosa relacionada a diagnósticos alternativos en estos pacientes, tal es el caso de la tromboembolia pulmonar o miocardiopatía, por mencionar algunos.

Debido a la evolución constante de las definiciones de caso sospechoso en la actual pandemia, la RSNA también ha publicado recientemente recomendaciones para mejorar las prácticas en los servicios de radiología con énfasis en la protección de los pacientes y personal del departamento de imagenología. Algunas de las pautas más relevantes incluyen la realización de TC de tórax diagnóstica en pacientes con alta probabilidad preprueba para neumonía por SARS-CoV-2 a pesar de tener RT-PCR negativa así como en pacientes con diagnósticos que requieran imagen de urgencia por otros motivos (apendicitis, colecistitis, evento vascular cerebral, etc.). Otros estudios de imagen no urgentes serán diferidos hasta que se obtengan los resultados de las pruebas de biología molecular con el afán de implementar las medidas de protección adecuadas según sea el caso. ${ }^{9}$

Los hallazgos de nuestro estudio corroboran la buena sensibilidad de la tomografía computarizada en el contexto de neumonía por SARS-CoV-2; sin embargo, su especificidad aún es cuestionable, y por ello no se le puede considerar el estándar de referencia en el diagnóstico de esta enfermedad. A pesar de todo lo anterior, las características de esta herramienta de imagen sí le permiten ser un recurso de posible utilidad para tamizaje y pronóstico en contextos clínicos adecuados.

La principal debilidad de nuestro estudio radica en el número limitado de pacientes incluidos al momento de la recolección de datos así como un posible sesgo

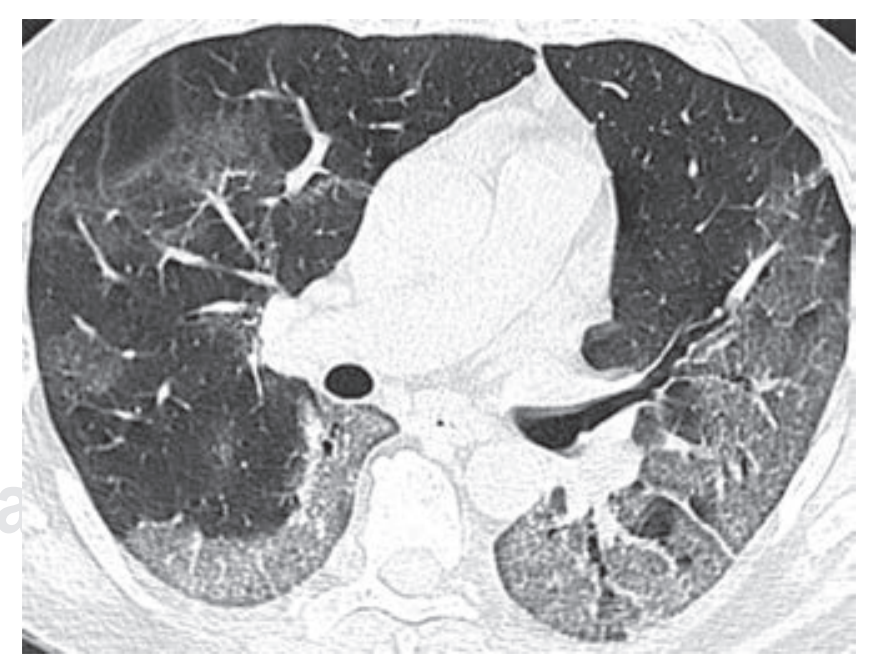

Figura 4: Patrón en crazy-paving bilateral y de distribución periférica asociado a múltiples zonas de vidrio despulido: hallazgos tomográficos típicos consenso RSNA/STR/ACR con categoría equivalente CO-RADS 5. 


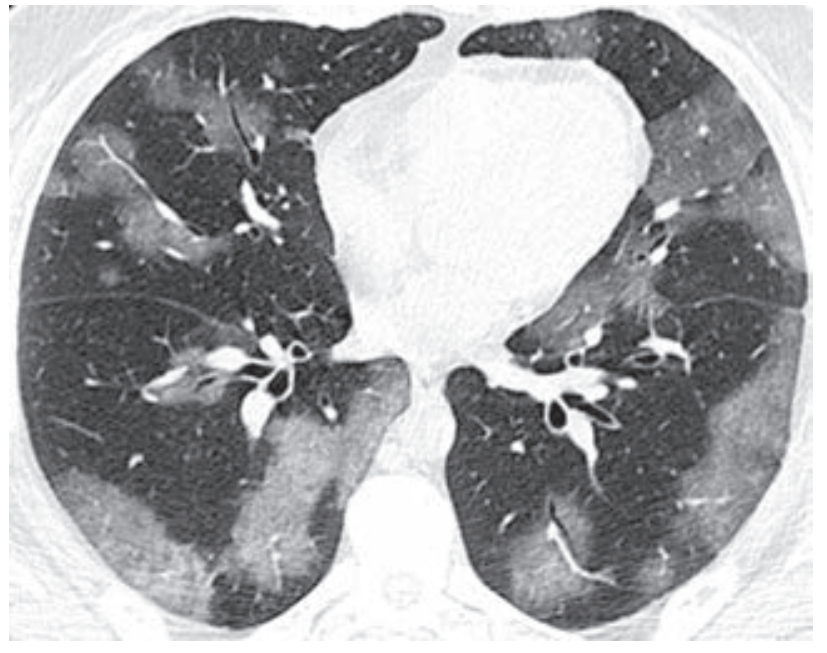

Figura 5: Múltiples infiltrados en vidrio despulido bilaterales y de distribución periférica: hallazgos tomográficos típicos consenso RSNA/STR/ACR con categoría equivalente CORADS 5.

de selección al ser un hospital privado donde se realizó nuestro estudio.

\section{CONCLUSIÓN}

El uso de tomografía computarizada en pacientes con sospecha o seguimiento por neumonía por SARS-CoV-2 ha de racionalizarse e individualizarse en cada centro hospitalario, teniendo en cuenta factores como la disponibilidad de recursos, traslado de pacientes, exposición del personal de salud, sanitización de áreas y equipos médicos, entre otros.

En nuestro centro hospitalario el papel de la tomografía ha recaído fundamentalmente en el manejo médico de los pacientes, lo anterior de forma similar a lo publicado por Rang Yang y colaboradores, ${ }^{10}$ ya que la severidad de afección pulmonar (volumen del parénquima pulmonar afectado) junto con datos clínicos dictamina las pautas para el ingreso a las unidades de cuidados intensivos, intermedios y hospitalización en área de aislamiento.

\section{REFERENCIAS}

1. COVID-19 Tablero México-CONACYT - CentroGeo - Geolnt - DataLab [Internet]. [Cited 2020 May 26]. Available from: https://coronavirus. gob.mx/datos/.

2. Tay MZ, Poh CM, Rénia L, MacAry PA, Ng LFP. The trinity of COVID-19: immunity, inflammation and intervention. Nat Rev Immunol. 2020; 1-12. Available from: http://www.nature.com/ articles/s41577-020-0311-8.

3. Simpson S, Kay FU, Abbara S, Bhalla S, Chung JH, Chung $M$ et al. Radiological Society of North America Expert Consensus Statement on Reporting Chest CT Findings Related to COVID-19. Endorsed by the Society of Thoracic Radiology, the American College of Radiology, and RSNA. Radiol Cardiothorac Imaging. 2020; 2 (2): e200152. Available from: http://pubs.rsna.org/doi/10.1148/ryct.2020200152.

4. Prokop M, van Everdingen W, van Rees Vellinga T, Quarles van Ufford J, Stöger L, Beenen L et al. CO-RADS-A categorical CT assessment scheme for patients with suspected COVID-19: definition and evaluation. Radiology. 2020; 296 (2): E97-E104. Available from: http://www.ncbi.nlm.nih.gov/pubmed/32339082.

5. Fuente-Alba C S, Molina VM. Likelihood ratio (razón de verosimilitud): definición y aplicación en radiología. Rev Argentina Radiol. 2017; 81 (3): 204-208.

6. Dawson GF. Interpretación fácil de la bioestadística: la conexión entre la evidencia y las decisiones médicas. Elsevier Health Sciences Spain; 2009. p. 205.

7. American College of Radiology. Revised 2018. 2018; 1-12. Available from: https://acsearch.acr.org/docs/69504/Narrative/.

8. Rubin GD, Haramati LB, Kanne JP, Schluger NW, Yim JJ, Anderson DJ et al. The role of chest imaging in patient management during the COVID-19 pandemic: a multinational consensus statement from the Fleischner Society. Radiology. 2020; 201365.

9. Mossa-Basha M, Azadi J, Ko J, Klein J, Meltzer C. RSNA COVID-19 task force: best practices for radiology departments during COVID-19. Radiological Society of North America. 2020; 1-7.

10. Yang R, Li X, Liu H, Zhen Y, Zhang X, Xiong Q et al. Chest CT severity score: an imaging tool for assessing severe COVID-19. Radiol Cardiothorac Imaging. 2020; 2 (2): e200047. Available from: http:// pubs.rsna.org/doi/10.1148/ryct.2020200047.

Conflicto de intereses y financiamiento: Héctor Manuel Velasco, Francisco René Hernández, Horacio Lozano, Mario Calva y Ángel César Chávez declaran que no tienen ningún conflicto de intereses. Financiamiento: ninguno. 